\title{
Why is the black hole entropy (almost) linear in the horizon area?
}

\author{
Gilad Gour* and Avraham E. Mayo ${ }^{\dagger}$ \\ The Racah Institute of Physics, Hebrew University of Jerusalem, \\ Givat Ram, Jerusalem 91904, Israel
}

(October 31, 2018)

\begin{abstract}
We show that essentially pure classical thermodynamics is sufficient to determine Bekenstein's formula for the black hole's entropy, $S=\eta A$. We base our reasoning on the minimal assumption that since black body radiation is describable by classical thermodynamics, so is the complete black hole-Hawking radiation system. Furthermore, we argue that any non-linear correction to the black hole entropy must be quantum mechanical in nature. The proportionality coefficient, $\eta=1 / 4 \ell_{P}^{2}$, must be calculated within a semi-classical or full-fledged quantum mechanical framework.
\end{abstract}

PACS number(s): 04.70.Dy, 04.70.-s 04.70.Bw

\section{INTRODUCTION}

It has been a quarter of a century since Bekenstein [1] and Hawking [2] suggested that the entropy of a black hole is one fourth of its surface area:

$$
S_{B H}=\frac{1}{4} \frac{A_{\mathcal{H}}}{\ell_{P}^{2}} .
$$

Despite considerable effort [3] on the quantum [4], dynamic [5], and statistical [6] origins of black hole thermodynamics, the exact source and mechanism of the Bekenstein-Hawking black hole entropy remain unclear [7]. By using the brick wall model, 't Hooft [8] identified the black hole entropy with the entropy of a thermal gas of quantum field excitations outside the event horizon, whereas Frolov and Novikov [4] argued that the black hole entropy can be obtained by identifying the dynamical degrees of freedom of a black hole with the states of all fields which are located inside the black hole. A black hole acts as classical thermodynamic object, but its true microscopic structure remains unclear 9].

One of classical thermodynamics' aspects is that the entropy and temperature of many systems can be derived within its framework without any need for referring to an underlying quantum theory. An outstanding example is the Stephen-Boltzmann formula for the energy and entropy flux densities of the radiation emitted by a hot black body 10

$$
u=\sigma T^{4}, \quad s=\frac{4}{3} \sigma T^{3} .
$$

Historically this formula was derived before the discovery of quantum mechanics. The coefficient $\sigma=\pi^{2} / 15 \hbar^{3}$ was calculated only after a quantum mechanical grasp of the phenomenon was achieved.

Reapplying this train of thoughts, we wish to establish here that the proportionality between black hole entropy and horizon area is a classical result. Any correction to the black hole entropy formula, $S=\eta A$, must be quantum mechanical in nature i.e. not derivable from classical thermodynamics. And just as it happened with $\sigma$, so here the coefficient $\eta=1 / 4 \ell_{P}^{2}$, must be calculated within a semiclassical or a quantum statistical framework [11.

Such an attempt has been made a few years ago by Gould [12], who claimed to establish the proportionality between black-hole entropy and horizon area based on the assumption that black holes are classical thermodynamics objects. After giving evidence for the relation $S=F(A)$ (to be rediscussed in Sec. II below), Gould uses the principle of equivalence to argue that the local temperature of radiation in the exterior of the black hole may depend only on the local gravitational "pull" felt by the local stationary observer. Accordingly, he concludes that the proportionality factor must be a universal constant of nature. A straightforward calculation shows that this proportionality factor is equal to $8 \pi F^{\prime}(A)$, and therefore that $F(A) \propto A$. However, it seems that Gould's argument is in not really classical. After all, the general connection between temperature and acceleration as put in evidence by Unruh [13], depends on quantum field theory.

In this work, we do recognize the quantum mechanical foundation from which Hawking radiation was derived, but also assume that inasmuch as black body radiation may be investigated using the tools of 19 th century classical thermodynamics, so may Hawking radiation.

The structure of the paper is as follows. We begin in Sec. II by reviewing the evidence for the relation $S=F(A)$, where $F$ is a positive definite and nondecreasing function. Bekenstein's historical argument for the form of the black hole entropy, $F(A) \propto A$ has evidently some logical gaps in it; it very much guesses the form of $F$. In Sec. III we try to bridge this gap. It is shown that classical thermodynamics decrees the relation $S \sim A^{\gamma}$, with $\gamma$ a constant. In Sec. IV, various physical arguments are used in order to explore this relation and constrain $\gamma$. The arguments are based upon various ideas at the base of classical thermodynamics, such as 
positive temperature for systems in equilibrium, negative specific heat for self-gravitating systems, and the generalized second law (GSL). We find that $\gamma>1 / 2$. Then, assuming that Hawking radiation can be described by geometrical optics (short wave approximation) we prove that $5 / 6<\gamma \leq 1$. In Sec. $\bigvee$ we present further arguments for why the relation $S \propto A(\gamma=1)$ should be singled out. Finally, the values of the proportionality coefficient and additive constant are discussed.

Throughout we assume that the system under investigation is embedded in a $3+1$ spacetime. The question concerning the possible nonlinear relation between black hole entropy and horizon area in higher dimensions, was addressed recently by Castro, Graniky and Naschie [14]. Furthermore, we deal only with bare black holes, excluding the so called "dirty" black holes, which are known to have a modified entropy formula due to the presence of classical fields on the horizon [15].

We use units with $\hbar=G=c=k_{B}=1$, unless otherwise stated.

\section{EVIDENCE FOR $S=F(A)$}

Our framework is classical thermodynamics. We shall not use quantum mechanics nor statistical mechanics. The black hole and surroundings are treated as a thermodynamical system in equilibrium with definite temperature $T$ and entropy $S$, which are quantities assumed to be definite functions of the black hole macroscopic parameters. In the spirit of the "no hair" theorem, these are the mass $M$, charge $Q$ and angular momentum $J$ of the black hole.

The first law of thermodynamics applied to the system black hole-surrounding may be written as

$$
d M=T d S-d \mathcal{W}
$$

where $\mathcal{W}$ is the work done on (or extracted from) the black hole. Specifically, let $\Phi$ and $\Omega$ be the electric potential and the angular velocity on the event horizon respectively. Then the work done (energy extracted) due to changes in angular momentum and electric charge is

$$
d \mathcal{W}=\Phi d Q+\Omega d J
$$

Substituting this in Eq. (3) gives

$$
T d S=d M-\Phi d Q-\Omega d J
$$

By exploring the dynamics of test objects in the black hole exterior, $\Phi$ and $\Omega$ are found to be 16

$$
\Phi=\frac{4 \pi r_{+} Q}{A}, \quad \Omega=\frac{4 \pi a}{A}
$$

where $r_{+}=M+\sqrt{M^{2}-Q^{2}-a^{2}}$ and $A=4 \pi\left(r_{+}^{2}+a^{2}\right)$ are the radius and area of the event horizon respectively, and $a \equiv J / M$ is the specific angular momentum.
We proceed by differentiating the horizon area $A$ with respect to $M, Q$ and $J$. The result is nothing but the first law of black holes mechanics:

$$
\frac{1}{8 \pi} \kappa d A=d M-\Phi d Q-\Omega d J
$$

where $\kappa$ is the surface gravity given by

$$
\kappa=8 \pi\left(\frac{\partial M}{\partial A}\right)_{J, Q}=\frac{4 \pi \sqrt{M^{2}-Q^{2}-a^{2}}}{A} .
$$

Combining now Eqs. (5) and (7) gives:

$$
\frac{1}{8 \pi} \kappa d A=T d S
$$

which impels us to infer that the black hole entropy must be a definite function of its horizon area:

$$
S=F(A)
$$

Gould [12] obtained the same result based on the argument that two black holes with the same horizon area must have the same entropy, since otherwise one would be able to violate the GSL by Penrose processes $[17$.

An interesting question is whether the result that $S=$ $F(A)$ is generic, namely, does it apply to others types of black holes besides the Kerr-Newmann black hole? The answer to this query seems to be on the affirmative. For example, a $(2+1)$-dimensional (BTZ) black hole obeys the standard first law of thermodynamics (5) augmented by an additional work term, $-\mathcal{P} d(2 \pi R)$, where $\mathcal{P}$ is the surface pressure at the boundary of a cavity of radius $R$. Accordingly, the same arguments which served to prove that the relation $S=F(A)$ for the Kerr-Newman black hole, may be used now to prove that the entropy of the BTZ black hole must be some function of its horizon area, and indeed, semi-classical calculations yields a linear entropy to horizon area relation [18]. Another example is the dilatonic black hole for which the entropy is proportional to the horizon area [19]. Keeping these other interesting cases in mind, we limit our analysis to the case of the Kerr-Newman black hole, since it captures the essentials of the ideas discussed in this work.

The result $S=F(A)$ clashes with a recent result by Vaz and Witten [20], who showed that in the framework of canonical quantum gravity the entropy of a charged black hole turns out to be the difference between the outer and inner horizon areas. Vaz and Witten explain this disagreement with the semi-classical result, as the product of neglecting the effect of back reaction to any radiation emitted exterior to the black hole. Another explanation involves a similar effect induced by emission of radiation in the interior of the black hole, radiation which is undetectable by an exterior observer. However, as Vaz and Witten admit themselves, their result may be due to the too restrictive boundary conditions imposed on the wave functional in the interior of the black hole 
(the wave functional is made to vanish beyond the inner horizon). However, they hedge this claim by pointing out that other boundary conditions are in effect unknown.

Keeping this in mind we proceed by noting that the black hole temperature is given by

$$
T=\left(\frac{\partial M}{\partial S}\right)=\frac{\kappa}{8 \pi F^{\prime}(A)}=\frac{\sqrt{M^{2}-Q^{2}-a^{2}}}{2 A F^{\prime}(A)} .
$$

Interestingly, for the black hole to have a non negative temperature, $F^{\prime}(A)>0$ i.e. $F(A)$ must be a monotonic non-decreasing function. In view of Hawking's increasing area theorem, this result had been expected, and indeed was assumed by Bekenstein [1]. In the rest of the paper we resort to classical thermodynamics to specify $F(A)$.

\section{INVESTIGATING $F(A)$}

Leaning on the parallelism between the zeroth law of black hole mechanics ( $\kappa$ is constant over the whole of the event horizon surface of a stationary black hole), and the zeroth law of thermodynamics ( $T$ is uniform over a system in equilibrium), one might conclude that the thermodynamical temperature must be a definite function of the surface gravity, and then invoke Eq. (11) to conclude that $F^{\prime}(A)=$ const, $F$ is linear in $A$, and the proof is complete.

However, there is a loophole: $T$ may also depend on $\Phi$ and $\Omega$, which are themselves constants on the horizon. Hence, it is less clear why $F$ should be linear in $A$. In order to go forward, we first prove the following statement:

The black hole entropy must have the form:

$$
S=\eta A^{\gamma}+S_{0}
$$

where $\eta$ and $S_{0}$ are constants of integration and $\gamma$ is a dimensionless parameter.

To prove this, we consider the Reissner-Nordstrom black hole (the derivation based on the Kerr solution is similar). Taking the logarithm in both sides of Eq. (11) and differentiating gives

$$
\frac{d T}{T}+\left(A \frac{F^{\prime \prime}(A)}{F^{\prime}(A)}+1\right) \frac{d A}{A}=\frac{M d M-Q d Q}{M^{2}-Q^{2}} .
$$

Consider now an isothermal process $(d T=0)$. Then

$$
1+A \frac{F^{\prime \prime}(A)}{F^{\prime}(A)}=\left.\frac{A}{M^{2}-Q^{2}} \frac{M d M-Q d Q}{d A}\right|_{T=\text { const. }}
$$

Using now Eq. (7) with $\Omega=0$, we find that

$$
\left.\frac{M d M-Q d Q}{d A}\right|_{T=\text { const. }}=\frac{\kappa}{8 \pi} \frac{M\left(\frac{\partial M}{\partial Q}\right)_{T}-Q}{\left(\frac{\partial M}{\partial Q}\right)_{T}-\Phi} .
$$

Substituting this and the expressions for $\Phi$ and $\kappa$ (Eqs. (6) and (8), respectively) in Eq. (14), and using the dimensionless parameter $y \equiv Q / M$, we find that

$$
\begin{aligned}
1+A \frac{F^{\prime \prime}(A)}{F^{\prime}(A)} & =\frac{1}{2 \sqrt{1-y^{2}}} \frac{\Phi_{T}-y}{\Phi_{T}-\frac{y}{1+\sqrt{1-y^{2}}}} \\
\Phi_{T} & \equiv\left(\frac{\partial M}{\partial Q}\right)_{T} .
\end{aligned}
$$

Keep in mind that $|y| \leq 1$, where equality is achieved for extremal black holes. $\Phi_{T}$ can be interpreted as the electric potential on the horizon when the black hole is in equilibrium with a surrounding heat bath. Since we are interested in the classical regime, we expand $\Phi_{T}$ in powers of $\hbar$ with the leading term, $O\left(\hbar^{0}\right)$ is taken to be classical. Higher order terms in $\hbar$ are considered to be quantum corrections. The classical leading term in $\Phi_{T}$ cannot vanish because in the limit $y \rightarrow 1$ the right hand side of Eq. (16) diverges unless $\Phi_{T} \rightarrow 1$. This may be anticipated, because in the limit of zero temperature (that is $y=1$ ) $\Phi_{T}$ should be equal to $\Phi$ because the black hole can be considered to be isolated from the thermal bath (i.e. $T=0)$. Hence, $\Phi_{T}(y=1)=\Phi(y=1)=$ 1. Accordingly, hereafter we assume that $\Phi_{T}$ has a non vanishing classical part.

Since $\Phi_{T}$ is a dimensionless quantity it must be a function of $y$ only. Consequently we have achieved separation of the variables in the problem, $y$ and $A$ : the right hand side of Eq. (16) is a function of $y$ only whereas the left hand side is a function of $A$ only. Since, $A$ and $y$ are two independent parameters this implies that

$$
1+A \frac{F^{\prime \prime}(A)}{F^{\prime}(A)}=\text { constant } \equiv \gamma .
$$

Solving this for $F(A)$ with $\gamma \neq 0$ reproduce Eq. (12). One must add that for $\gamma=0$, the solution is logarithmic in $A$. However in the next section we explain why this possibility must be excluded.

\section{CONSTRAINING $\gamma$.}

Here we use various arguments to constrain $\gamma$. In subsection IVA we use some basic principles of classical thermodynamics to set the constrain $\gamma>1 / 2$. This result is of great importance since it emphasizes the fact that black hole thermodynamics is not like ordinary thermodynamics. Surely the choice $\gamma=1 / 2$, e.g. $S \sim \sqrt{A}$, seems to stands out because it implies that for $Q=J=0, S \propto M$, in harmony with the extensive character of entropy in ordinary thermodynamics. But as proven below, this later choice clashes with basic principles of black holes and traditional physics, and thus should be rejected.

Consider now, Hawking radiation. Obviously, Hawking's celebrated derivation of the black hole radiation 
is quantum-mechanical. Consequently it could be argued that by considering this phenomenon we are actually invalidating our claim that the derivation of black hole entropy may be based on purely classical arguments. But in a sense thermodynamics is sensitive to effects at the quantum level; while classical mechanics is consistent with the limit $\hbar \rightarrow 0$, classical thermodynamics is not. Accordingly we make the minimal assumption that the laws of thermodynamics apply to the black hole and radiation as a complete system (after all, if the black hole is to be considered a thermodynamical object with entropy and temperature, it must be able to radiate as well as absorb). This implies that the radiation may be described by the Stephan-Boltzmann law. As explained in subsection IVB this leads us to conclude that $5 / 6<\gamma \leq 1$.

It should be pointed out that in his article, Gould does not address the issue of Hawking radiation and the impact it may have on the derivation of the black hole entropy formula. Hawking himself discusses it in [2].

\section{A. Positive temperature, Negative Specific Heat and the GSL: $\gamma>1 / 2$.}

Consider first the temperature of the Schwarzschild black hole Eq. (11),

$$
T=\frac{M}{2 A F^{\prime}(A)}=\frac{1}{2 \eta \gamma(16 \pi)^{\gamma} M^{2 \gamma-1}} .
$$

The first obvious fact is that $\gamma$ must be positive definite for the system to have positive definite temperature. Examining next the specific heat $(\partial T / \partial M)^{-1} \propto 1-2 \gamma$; we find that for the system to have a negative specific heat, as befits a self-gravitating system, $\gamma$ must be greater then $1 / 2$. Note that if the logarithmic solution of Eq. (17) were to be taken seriously, it would imply that the black hole temperature is linear in the mass, and hence the specific heat would be constant independent of the black hole mass.

A somewhat related argument supporting the constraint $\gamma>1 / 2$, involves the interplay between the gravitational and the electric forces in the physics of a Reissner-Nordstrom black hole. It is reasonable to assume that when the black hole charge is small, the thermodynamics of the system is governed primarily by gravitation, which decrees a negative specific heat. However, as the magnitude of the black hole charge is increased, electrodynamics becomes important. At some point the system should begin to show typical qualities of a traditional thermodynamical system, such as a positive specific heat. Consequently, at some critical values of $Q$ and $M$, the specific heat should switch sign from negative to positive through a second order phase transition. For example, for $\gamma=1$ this transition takes place when $Q=\sqrt{3 / 4} M$. For a general $\gamma$ this condition translates to $Q=\sqrt{1-1 /\left(4 \gamma^{2}\right)} M$. If this phenomenon is to be reproduced, $\gamma$ must again be greater than $1 / 2$.
A more rigorous proof for $\gamma>1 / 2$ can be drawn from the following gedanken experiment. Consider two identical Schwarzschild black holes of mass $M$, which collide and merge to form a third black hole of mass $\mathcal{M}$. The initial entropy of the system is $S_{i}=2 \eta\left(16 \pi M^{2}\right)^{\gamma}+2 S_{0}$. During the process of in-spiraling and finally coalescence, the system lose energy primarily by emission of gravitational waves and negligibly by thermal radiation. Accordingly the mass of the black hole at the end state of the system must be smaller then the initial energy contained in the two well separated black holes: $\mathcal{M}<2 M$. It follows that the entropy at the end state of the system, $S_{f}$ is bounded from above by $\eta\left(64 \pi M^{2}\right)^{\gamma}+S_{0}$. But by the GSL it must also be greater then the entropy at the initial state of the system. Combining these two arguments gives

$$
2 \eta\left(16 \pi M^{2}\right)^{\gamma}+2 S_{0}<S_{f}<\eta\left(64 \pi M^{2}\right)^{\gamma}+S_{0} .
$$

The only way in which this can be true for arbitrary $M$ is for

$$
\gamma>1 / 2
$$

As a last remark we point out that this gedanken experiment also provide us with clear cut evidence against $F(A)=\ln A$, since it implies that the GSL would be violated for $A>4 \exp \left(-S_{0} / \eta\right)$ (note that here $S_{0}$ and $\eta$ are both dimensionless).

\section{B. Hawking radiation: $5 / 6<\gamma \leq 1$.}

Assume now that the radiation emitted by a Schwarzschild black hole follows the Stephan-Boltzmann law (2). We are clearly assuming that geometrical optics may be applied here, that is, that the characteristic wavelength of the radiation $\lambda_{\max }$, is smaller than the characteristic length scale of the emitter, namely $2 M$. In the regime where $\lambda_{\max } \gg M$ geometrical optics ceases to provide a good description for the radiation, and wave optics must be employed instead. However, since $\gamma$ is a constant of the theory, any result concerning $\gamma$ which was obtained within a regime describable by geometrical optics, should be extendable to regimes where geometrical optics fails.

Keeping this point in mind, we recall that by the principle of black body radiation, the radiated power (which equals minus the rate of change of the black hole mass) is given by

$$
\dot{M} \propto-T^{4} A \propto-M^{2(3-4 \gamma)} .
$$

In the limit $M \rightarrow \infty$, the temperature approaches zero and thus should also the radiated power $\dot{M}$. This indicate that $\gamma$ must be bigger than $3 / 4$. Furthermore, the rate of change of the black hole entropy is

$$
\dot{S}=\frac{\dot{M}}{T} \propto-M^{5-6 \gamma} .
$$


In the limit where $T \rightarrow 0(M \rightarrow \infty), \dot{S} \rightarrow 0$, which implies that $\gamma>5 / 6$. Also, combining Eqs. (22) and (21), one finds that

$$
\dot{S} \propto \dot{M}^{\frac{5-6 \gamma}{2(3-4 \gamma)}} .
$$

It is reasonable to assume that $\dot{S}$ and $\dot{M}$ increase (decrease) simultaneously. Accordingly, we infer that (5$6 \gamma) /(3-4 \gamma)>0$. Since it was already shown that $\gamma>3 / 4$, the constraint $\gamma>5 / 6$ is reproduced.

Consider next Wein's law which teaches us that

$$
\lambda_{\max } T \sim \hbar,
$$

where $\lambda_{\max }$ corresponds to the wavelength at which the radiation intensity is maximized. This suggests that $\lambda_{\max } \sim 1 / T \sim M^{2 \gamma-1}$. Recalling now that this would be true provided that

$$
\frac{\lambda_{\max }}{2 M} \sim M^{2(\gamma-1)} \lesssim 1
$$

we accept that $\gamma \leq 1$. Thus we conclude that

$$
5 / 6<\gamma \leq 1
$$

\section{ARGUMENTS FOR $\gamma=1$. WHAT CAN BE SAID ABOUT $S_{0}$ AND $\eta$ ?}

The first argument is based on the observation that the power by which the temperature is raised in the StephanBoltzman law is determined by the effective dimension of the phase space by which the system is described. The rule that seems to emerge is that the power by which the temperature is raised is equal to $\mathcal{D}+1$, where $\mathcal{D}$ is the effective dimension of the system. Keeping this in mind, we rewrite the expression for the radiated power (21) in term of the temperature

$$
\dot{M} \propto-T^{4} A \propto-T^{4-\frac{2}{2 \gamma-1}} .
$$

This suggests that the black hole is in effect a thermodynamic system with an effective dimension $\mathcal{D}$, fixed by the equality

$$
\mathcal{D}+1=4-\frac{2}{2 \gamma-1} .
$$

The constraint on $\gamma$ may be used now to constrain $\mathcal{D}$ and even to tell us something about $\gamma$. Solving Eq. (28) for $\gamma$ we find

$$
\gamma=\frac{1}{2}+\frac{1}{3-\mathcal{D}}
$$

Using the result of the previous section, $5 / 6<\gamma \leq 1$, we infer that $0<\mathcal{D} \leq 1$. Assuming that $\mathcal{D}$ is an integer (glossing over the possibility of fractal dimensions which is usually an attribute of systems lacking any kind of characteristics scale), we find that $\gamma$ may have only one possible value,

$$
\gamma=1 .
$$

Remarkably, even though the black hole is evidently a $3 D$ object embedded in a $3+1$ dimensional spacetime, it behaves as if it was a one dimensional thermodynamical object!

Imagine now a number of black holes in vacuum momentarily at rest at some distance one from another [21]. The only evident source of entropy is the black holes horizons. Presuming that the entropies of independent systems are additive, we may write

$$
S_{\text {total }}=\sum_{i}\left(\eta A_{i}^{\gamma}+S_{0}\right) .
$$

We make the assumption that the entropy contributed by a specific black hole is not changed by its motion and associated dynamical changes, induced by its companions, so that the formula for $S_{\text {total }}$ applies also when the black holes falls towards each other. Focus now on two of these black holes, with areas $A_{1}$ and $A_{2}$, as they fall towards each other and merge into a single one. By Hawking's result each $A_{i}$ is bound to increase [22]. Furthermore, the growth of the event horizon area is a continuous process (to be contrasted with the growth of the apparent hori$z o n$ area which may be discontinues). Therefore, at the moment of coalescence the horizon area of the new black hole is the sum of the areas of the merging constitutes, $A_{\text {new }}=A_{1}+A_{2}$. By the assumption that the relation between entropy and area is still valid in the case of distorted black holes, we may use Eq. (31) for the new black hole. During the infall and the merger processes, semicoherent gravitational radiation is emitted, which should not have much effect on the entropy balance. Hence at the moment of merger,

$$
\left(A_{1}+A_{2}\right)^{\gamma}=A_{1}^{\gamma}+A_{2}^{\gamma}+S_{0} / \eta .
$$

Obviously one can arrange for the process to occur with $A_{1}=A_{2}=A_{0}$, for example by slowly lowering two black holes of equal masses towards each other, so that the area increase and distortion are identical in both black holes. This must be true for any arbitrary $A_{0}$. So we are led to the conclusion that $\gamma=1$ and $S_{0}=0$. This agree with the reasonable assumption that the entropy tends to zero as the mass tends to zero. Moreover, the vanishing of the additive coefficient $S_{0}$ indicates that the zero of the entropy is set, without the liberty of adding a constant, as one can do in classical physics.

What can be said about the proportionality coefficient $\eta$ ? Obviously, $\eta$ should have dimensions of inverse area. Further insight seems to require the use of quantum physics. However, Wheeler's heuristic suggestion that the right order of magnitude of $S$ should be gotten by just dividing $A$ by the Planck length squared $\ell_{P}^{2}$, 
gained support from the observation by Bekenstein [1] that when an elementary particle is very softly deposited at the horizon of any Kerr-Newmann black hole, the minimal increase of $S$ so calibrated is of order unity. Since an elementary particle should carry no more than a unit of entropy, the GSL would fail if we took $\eta$ as an inverse length square with a length large on the Planck scale. Also it makes no sense to take this length scale to be smaller than Planck's length, which is regarded as the smallest scale on which smooth spacetime is a reasonable paradigm. Nothing could be said about the numerical magnitude of $\eta_{0} \equiv \eta \ell_{P}^{2}$ without over-stepping the realm of classical thermodynamics.

Thus we close by writing

$$
S_{B H}=\eta_{0} \frac{A_{\mathcal{H}}}{\ell_{P}^{2}} .
$$

ACKNOWLEDGMENTS It is a pleasure to thank Prof. J. D. Bekenstein for suggesting this problem and for his guidance during this work. This research is supported by a grant from the Israel Science Foundation, established by the Israel Academy of Sciences and Humanities.

[1] J. D. Bekenstein, Phys. Rev. D 7, 2333 (1973); Phys. Rev. D 9, 3292 (1974).

[2] S. W. Hawking, Commun. Math. Phys. 43, 199 (1975); Phys. Rev. D 13, 191 (1976).

[3] J. D. Bekenstein, in the Proceedings of the seventh Marcel Grossmann Meeting on Genral Relativity, (World Scientific, Singapore, 1994).

[4] L. Bombelli, R. Koul, J. Lee, and R. Sorkin, Phys. Rev. D 34, 373 (1986).

[5] V. Frolov and I. Novikov, Phys. Rev. D 48, 4545 (1993); V. Frolov, Phys. Rev. Lett. 74, 3319 (1995).

[6] H. Zurek and K. S. Thorne, Phys. Rev. Lett. 54, 2171 (1985).

[7] M. H. Lee and J. Kim, hep-th/9604130;Phys. Lett. A 212 , 323 (1996); J. Ho, W. T. Kim, Y. J. Park, and H. Shin, Class. Quant. Grav. 14, 2617 (1997).

[8] G. 't Hooft, Nucl. Phys. B 256, 727 (1985); L. Susskind and J. Uglum, Phys. Rev. D 50, 2700 (1994).

[9] M. Maggiore, Nucl. Phys. B 429, 205 (1994).

[10] The textbook proof goes as follows. Let us make a blackbody enclosure with a piston, so that work may be done on or extracted from the radiation. By the first law of thermodynamics, we have $d Q=d U+p d V$, where $Q$ is heat and $U$ is total energy. By the second law of thermodynamics $d S=d Q / T$, where $S$ is the entropy. Since $U=u(T) V$, and $p=u(T) / 3$, we have $d S=(V / T) u^{\prime}(T) d T+(4 u(T) / 3 T) d V$. Further, $d S$ is a perfect differential, thus we obtain

$$
\frac{1}{T} u^{\prime}(T)=\frac{\partial^{2} S}{\partial T \partial V}=-\frac{4 u(t)}{3 T^{2}}+\frac{4}{3 T} u^{\prime}(T),
$$

so that $u^{\prime}(t)=4 u(T) / T$ and thus $u(T)=a T^{4}$, where $a$ is a constant of integration. Thus we obtain the StephanBoltzmann law.

[11] for a comprehensive review see for example R. M. Wald, "Black Hole Thermodynamics" to appear in Living Reviews in Relativity, gr-qc/9912119.

[12] A. Gould, Phys. Rev. D 35, 449 (1987).

[13] W. G. Unruh, Phys. Rev. D 14, 870 (1976).

[14] C. Castro, A. Graniky and M.S.El Naschie, hepth/0004152

[15] V. Iyer and R. M. Wald, Phys. Rev. D 50, 846 (1994); Phys. Rev. D 52, 4430 (1995); M. Visser, Phys. Rev. D 48, 5697 (1993); Phys. Rev. D 48, 583 (1993)

[16] C. W. Misner, K. S. Thorne and J. A. Wheeler, Gravitation (Freeman, San Francisco, 1973).

[17] R. Penrose and R.M. Floyd, Nature Phys. Sci. 229, 177 (1971); D.Christodoulou and R. Ruffini, Phys. Rev. D 4, 3552 (1971).

[18] S. Carlip, Class. Quant. Grav. 122853 (1995).

[19] For a review on lower dimensional black holes see R.B. Mann, gr-qc/9501038.

[20] C. Vaz and L. Witten, gr-qc/0006039.

[21] J. D. Bekenstein, private communication.

[22] S. W. Hawking, Phys. Rev. Lett. 26, 1344 (1971). 